\title{
DELIMITAÇÃO AUTOMÁTICA DE BACIAS HIDROGRÁFICAS UTILIZANDO DADOS SRTM
}

\author{
TEODORICO ALVES SOBRINHO ${ }^{1}$, PAULO T. S. OLIVEIRA ${ }^{2}$, \\ DULCE B. B. RODRIGUES ${ }^{3}$, FABIO M. AYRES ${ }^{4}$
}

\begin{abstract}
RESUMO: A delimitação adequada de bacias hidrográficas é fundamental por ser essa a unidade territorial para fins de planejamento e de gerenciamento dos recursos hídricos. Desse modo, este trabalho teve objetivo de analisar uma metodologia de delimitação automática de bacias hidrográficas. Foram utilizados, para tanto, dados do projeto Shuttle Radar Topographic Mission (SRTM) integrados e processados em Sistema de Informações Geográficas (SIG). Os resultados confirmam valores de área compatíveis aos obtidos com base em cartas topográficas. Assim, a automatização da delimitação de bacias hidrográficas, por meio de dados do SRTM e ambiente SIG, apresenta-se vantajosa em relação ao custo e benefício proporcionado, além de estabelecer a padronização do traçado e posterior minimização de conflitos quanto à fixação da unidade de gestão dos recursos hídricos.
\end{abstract}

PALAVRAS-CHAVE: gestão de recursos hídricos, bacia hidrográfica, delimitação automática.

\section{AUTOMATIC DELIMITATION OF WATERSHED USING DATA SRTM}

\begin{abstract}
The adequate delimitation of watersheds is essential to be that the territorial unit for planning and management of water resources. Thus, this work aims to evaluate a methodology for automatic delimitation of drainage basins. The project Shuttle Radar Topographic Mission (SRTM) data were integrated and processed in the Geographic Information System (GIS). The results show values of area compatible to those obtained on the topographic data. So, the automation of delimitation of watersheds, through SRTM data and GIS environment, appears to be advantageous in relation to cost and benefits offered, in addition, permits the standardization of the sketch and minimization of conflicts during fixation of the elementary unit of water resources management.
\end{abstract}

KEYWORDS: watershed, automatic delimitation, water resource management.

\section{INTRODUÇÃO}

No Brasil, a Lei Federal no 9.433/97 estabelece a bacia hidrográfica como unidade territorial para aplicação da Política Nacional de Recursos Hídricos (PNRH). A fixação dessas unidades básicas envolve a abrangência de aplicação dos instrumentos da PNRH, tais como: enquadramento dos corpos d'água, outorga e cobrança pelo uso de recursos hídricos. Assim, padronização e automatização do traçado de bacias hidrográficas são fundamentais para a efetivação adequada da PNRH, evitando-se possíveis conflitos de utilização dos recursos hídricos.

O desenvolvimento e o aperfeiçoamento de técnicas de delimitação automática de bacias hidrográficas têm sido objeto de estudo em várias partes do mundo. Tais técnicas são implementadas em ambientes de Sistemas de Informações Geográficas (SIG), promovendo resultados relevantes, conforme verificado nos trabalhos de MARK (1984), O'CALLAGHAN \& MARK (1984), BAND (1986), JENSON \& DOMINGUE (1988), TARBOTON et al. (1991),

\footnotetext{
${ }^{1}$ Eng ${ }^{\circ}$ Agrônomo, Prof. Dr. Associado II, Departamento de Hidráulica e Transportes, Universidade Federal de Mato Grosso do Sul, Caixa Postal 549, Campo Grande - MS, talves@ufms.br. Bolsista do CNPq-PQ 2.

${ }^{2}$ Eng ${ }^{\circ}$ Ambiental, Mestrando em Saneamento Ambiental e Recursos Hídricos pela Universidade Federal de Mato Grosso do Sul, Campo Grande - MS, paulotarsoms@ gmail.com. Bolsista de Mestrado FUNDECT.

${ }^{3}$ Eng $^{\circ}$ Ambiental, Mestranda em Saneamento Ambiental e Recursos Hídricos pela Universidade Federal de Mato Grosso do Sul, , Campo Grande - MS, dulcebbr@gmail.com. Bolsista de Mestrado FUNDECT.

${ }^{4}$ Geógrafo, Prof. Mestre, Universidade Católica Dom Bosco, Campo Grande - MS, fabioayres@ @otmail.com.

Recebido pelo Conselho Editorial em: 25-8-2008

Aprovado pelo Conselho Editorial em: 20-11-2009

Eng. Agríc., Jaboticabal, v.30, n.1, p.46-57, jan./fev. 2010
} 
FAIRFIELD \& LEYMARIE (1991), VERDIN \& VERDIN (1999), TURCOTTE et al. (2001), VOGT et al. (2003), JORDAN \& SCHOTT (2005) e MERKEL et al. (2008).

No processo de delimitação automática de bacias hidrográficas em SIGs, são utilizadas informações de relevo, que podem ser representadas por uma estrutura numérica de dados correspondente à distribuição espacial da altitude e da superfície do terreno, denominada Modelo Numérico de Terreno (MNT). O MNT pode ser obtido por meio da interpolação de curvas de nível extraídas de uma carta topográfica ou através de imagens de sensores remotos.

JENSON \& DOMINGUE (1988) afirmam que parâmetros hidrológicos extraídos de MNTs mostram-se acurados e compatíveis com aqueles obtidos por métodos manuais, que despendem de maior tempo no seu processamento e têm detalhamento menor na sua configuração. TARBOTTON et al. (1991) e WALKER \& WILGOOSE (1999) descrevem que o MNT apresenta boa correlação entre a declividade e a área de contribuição, exibindo os pontos de inflexão que marcam o início da captação fluvial, de modo que a rede de drenagem pode ser determinada com confiança elevada.

Os MNTs produzidos a partir de informações de sensoriamento remoto, tal como o Interferometric Synthetic Aperture Radar (InSAR), têm ganho relevância por serem advindos de uma técnica rápida e acurada de coletar dados topográficos (RABUS et al., 2003). O sistema InSAR possibilita a obtenção de informações sobre as variações de altitude da superfície terrestre, sendo aplicável a diversos estudos relacionados à geomorfologia, análise de rede hidrográfica, delimitação de áreas inundáveis, perfis topográficos e delimitação automática de bacias hidrográficas, dentre outros.

A missão Shuttle Radar Topography Mission (SRTM), realizada em 2000 a bordo da nave Endeavour, incorpora a técnica InSAR e dispõe publicamente seus dados em escala global por intermédio do United States Geological Survey (USGS). Assim, diversas pesquisas vêm sendo conduzidas com o objetivo de analisar, comparar e atualizar informações da superfície terrestre por meio de dados do SRTM. Nesse contexto, podem-se citar os trabalhos desenvolvidos por DIAS et al. (2004), SANTOS et al. (2006), VALERIANO \& ABDON (2007), LUEDELING et al. (2007), FREDRICK et al. (2007), BERRY et al. (2007) e RENNÓ et al. (2008).

GERSTENECKER et al. (2005), avaliando várias bases para geração de MNTs, inclusive cartas topográficas, concluíram que a missão SRTM é um passo de importância considerável no detalhamento acurado dos MNTs do globo terrestre. PINHEIRO (2006) concluiu que as altitudes medidas pelo MNT obtido dos dados do SRTM, após as devidas correções, apresentaram resultados melhores, comparados com os MNTs gerados a partir de cartas topográficas em escala 1:50.000.

Desse modo, este trabalho teve como objetivo a análise de uma metodologia para delimitação automática de bacias hidrográficas, utilizando, para tanto, dados do projeto SRTM, integrados e processados em ambiente SIG, validados na bacia hidrográfica do Córrego Guariroba.

\section{MATERIAL E MÉTODOS}

O estudo foi realizado tomando-se por base a Área de Proteção Ambiental (APA) do Guariroba, criada em 1995 pelo Decreto $\mathrm{n}^{\circ}$ 7.183, abrangendo todo o limite da bacia hidrográfica do Córrego Guariroba. Essa bacia contém um dos principais mananciais de água superficial de Campo Grande - MS, responsável pelo abastecimento de aproximadamente $52 \%$ da população urbana do município. A localização espacial da bacia hidrográfica do Guariroba está representada na Figura 1. 


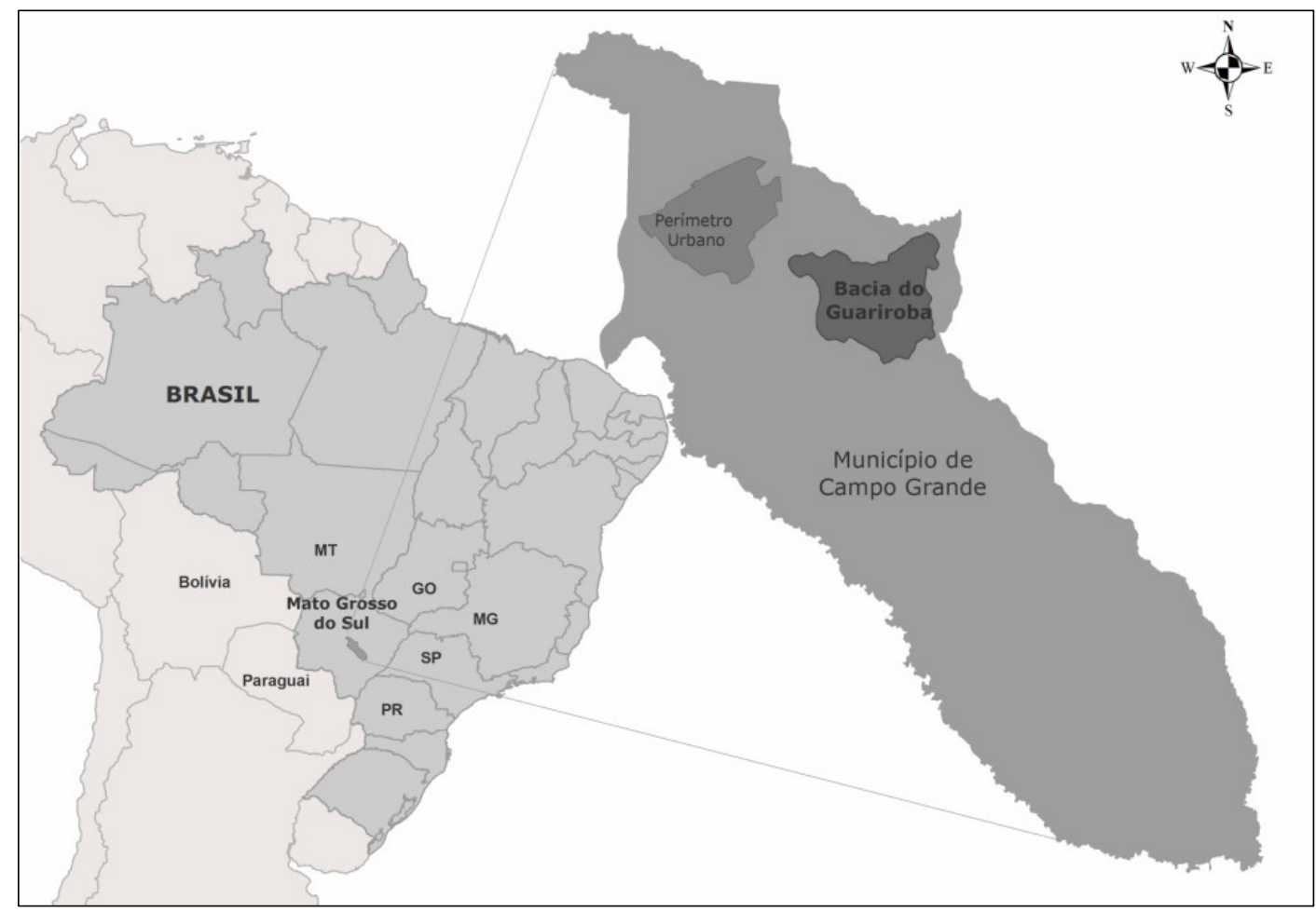

FIGURA 1. Mapa de localização da bacia do Córrego Guariroba. Location map of Guariroba river basin.

A bacia do Córrego Guariroba possui extensão de aproximadamente 37.000 ha e situa-se entre

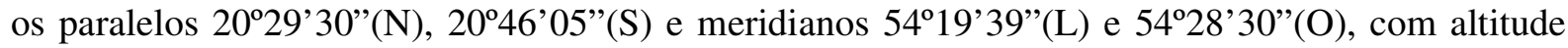
variando de $440 \mathrm{~m}$ a $640 \mathrm{~m}$. Está localizada na grande unidade geológica denominada Bacia Sedimentar do Paraná e encontra-se inserida na sub-bacia hidrográfica do Rio Pardo.

De acordo com o projeto RADAMBRASIL (BRASIL, 1982), a bacia hidrográfica do Guariroba encontra-se geomorfologicamente inserida no Planalto de Maracaju - Campo Grande, possuindo superfície de aplanamento, elaborada por processos de pediplanação, cortando litologias pré-cambrianas do Grupo Cuiabá e Corumbá, rochas devonianas e permocarboníferas da Bacia Sedimentar do Paraná. Os relevos de topo convexo, com diferentes ordens de grandeza e de aprofundamento de drenagem, são separados por vales de fundo plano ou em forma de "V".

Os dados do SRTM da região de estudo foram obtidos junto ao USGS, possuindo informações originais disponíveis para a América do Sul, referentes à banda $\mathrm{C}$ do equipamento InSAR, com resolução espacial de $90 \mathrm{~m}$ e elipsoide de referência WGS84. Assim, realizou-se a conversão para o Datum South American Datum (SAD 1969) com o auxílio do software ENVI 4.3, de modo a preparar os dados para posterior aplicação no trabalho.

O processo de delimitação automática da bacia hidrográfica foi desenvolvido no SIG ArcGIS (ESRI, 2006), juntamente com as extensões ("plugins") disponíveis no endereço eletrônico da Environmental Systems Research Institute (ESRI): Spatial Analyst e Hydrology Modeling.

A metodologia utilizada nesse processo subdividiu-se em quatro etapas, sendo: preenchimento de depressões ("fill sinks"), direção de fluxo ("flow direction"), fluxo acumulado ("flow accumulation”) e delimitação de bacias ("Watershed”) (DIAS et al., 2004) (Figura 2). 


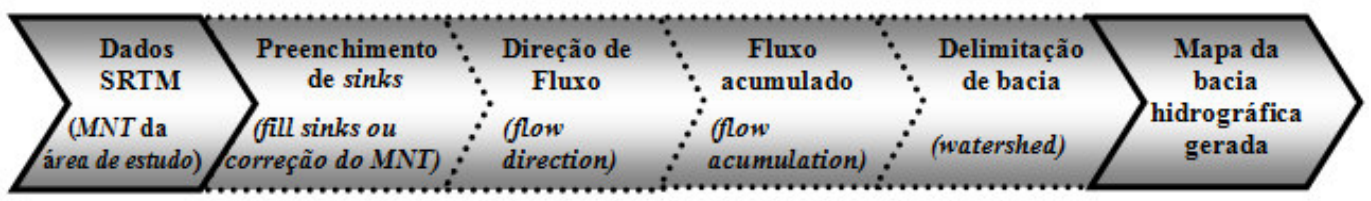

FIGURA 2. Principais etapas realizadas para delimitação de bacias hidrográficas a partir de dados SRTM. Main steps achieved for delimitation of watershed from SRTM data.

\section{Preenchimento de depressões ("fill sinks")}

A acurácia dos dados do SRTM tem sido comprovada de diversas formas por vários pesquisadores (JARVIS et al., 2004; KOCAK et al., 2005; RODRIGUEZ et al., 2006; SMITH \& SANDWELL, 2003; SUN et al., 2003). No entanto, estudos desenvolvidos por FALORNI et al. (2005) e KÄ̈̈B (2005) apontam que a acurácia dos dados do SRTM depende da topografia local, constatando-se maior incidência de erros em dados referentes a terrenos com relevo íngreme ou montanhoso.

Infelizmente, o MNT que acompanha os dados do SRTM contém falhas em áreas do globo, originadas, principalmente, de duas maneiras: ocorrência de corpos hídricos e relevo acidentado. Nessa última, a frequência de falhas é maior em superfícies com inclinação acima de $20^{\circ}$, devido ao sombreamento ocasionado no radar (LUEDELING et al., 2007).

As falhas no MNT advindas dos dados do SRTM são denominadas de "sinks" que, conforme MENDES \& CIRILO (2001), caracterizam-se por áreas rodeadas por elevações com valores de cotas superiores, semelhantes a uma depressão. O preenchimento dessas pequenas depressões é o primeiro tratamento dado à matriz de altitudes (Figura 3). Essas depressões ou "sinks" são consideradas empecilhos ao escoamento durante a aplicação de modelos hidrológicos, sedimentológicos e de poluentes de origem difusa.

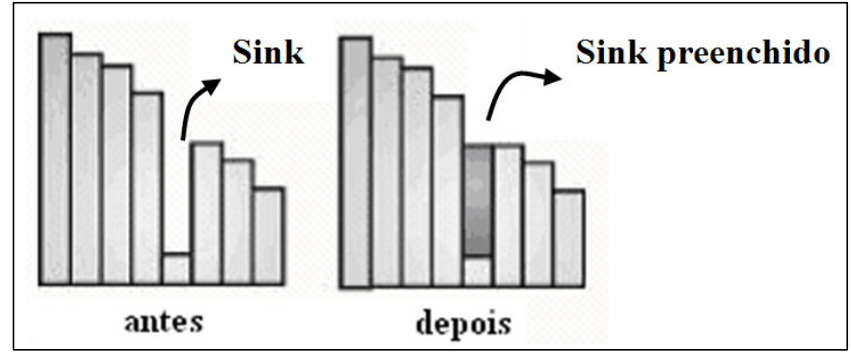

FIGURA 3. Correção de erros do tipo "sink" por meio da execução da função "fill sinks". Correction of errors type sink through the execution of the function fill sinks.

Conforme a Figura 3, as correções no MNT são feitas por meio da função "fill sinks", que considera as altitudes dos "pixels" vizinhos para preencher os "sinks", promovendo, assim, a geração do mapa de MNT com consistência melhor, tal como exposto na Figura 4. 


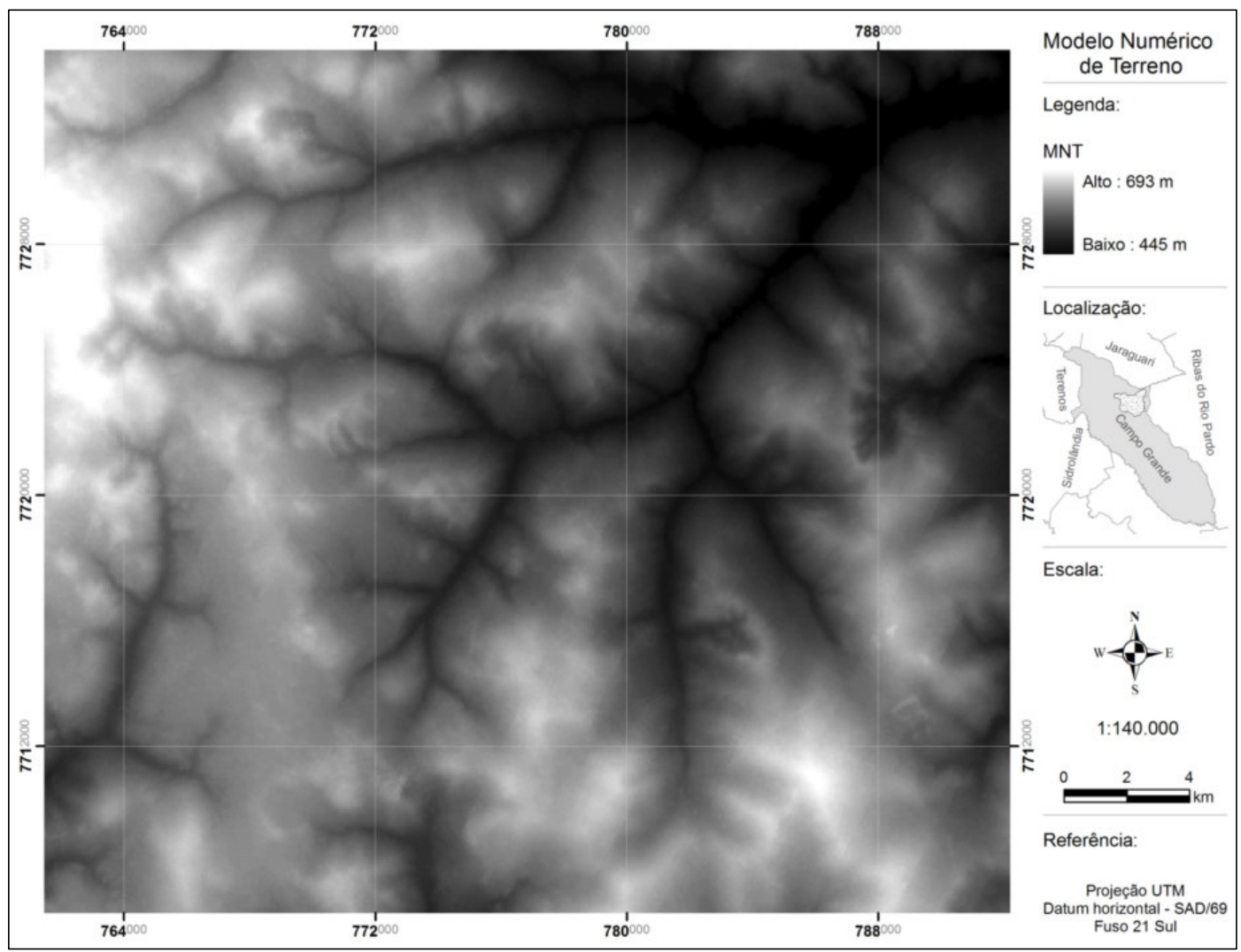

FIGURA 4. Mapa do MNT obtido do tratamento de dados SRTM. Map of MNT obtained from SRTM data processing.

\section{Direção de fluxo}

De acordo com RENNÓ et al. (2008), a direção de fluxo define as relações hidrológicas entre pontos diferentes dentro de uma bacia hidrográfica. A continuidade topológica para as direções de fluxo é, consequentemente, necessária para que uma drenagem funcional possa existir. As conexões hidrológicas de direção de fluxo entre dois pontos em uma superfície não são as mesmas que aquelas baseadas em distância Euclidiana.

A direção de fluxo de água na rede de drenagem é obtida pela função "flow direction", que gera uma grade regular definindo as direções de fluxo, tomando-se por base a linha de maior declividade do terreno.

A nova grade numérica gerada determina a direção de maior declividade de um "pixel" em relação a seus oito "pixels" vizinhos. Assim, ocorre a descrição numérica da direção que a água irá percorrer após atingir cada "pixel", que pode ser representada graficamente por meio da aplicação do código de direção (Figura 5).

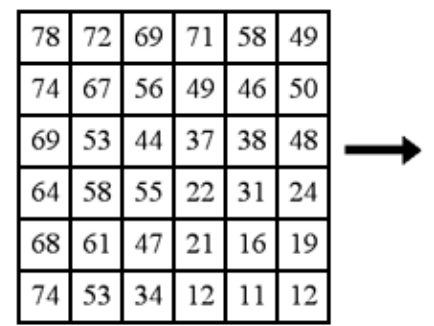

Elevação ou MNT

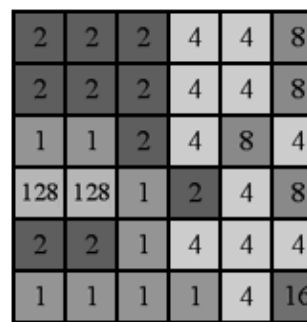

Direção de fluxo (forma numérica)

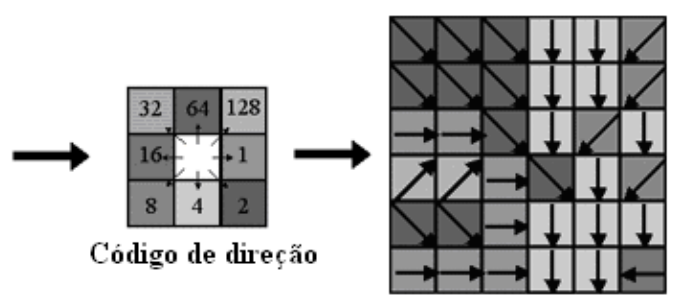

Direção de fluxo

FIGURA 5. Exemplo de execução da função "flow direction". Example of execution of flow direction function. Fonte: Adaptado de ESRI (2008). 
A execução da função "flow direction" com o MNT já corrigido na etapa anterior fornece o mapa de direção de fluxo apresentado na Figura 6, que possibilita a observação da direção do escoamento de água nas vertentes, além da visualização do relevo.

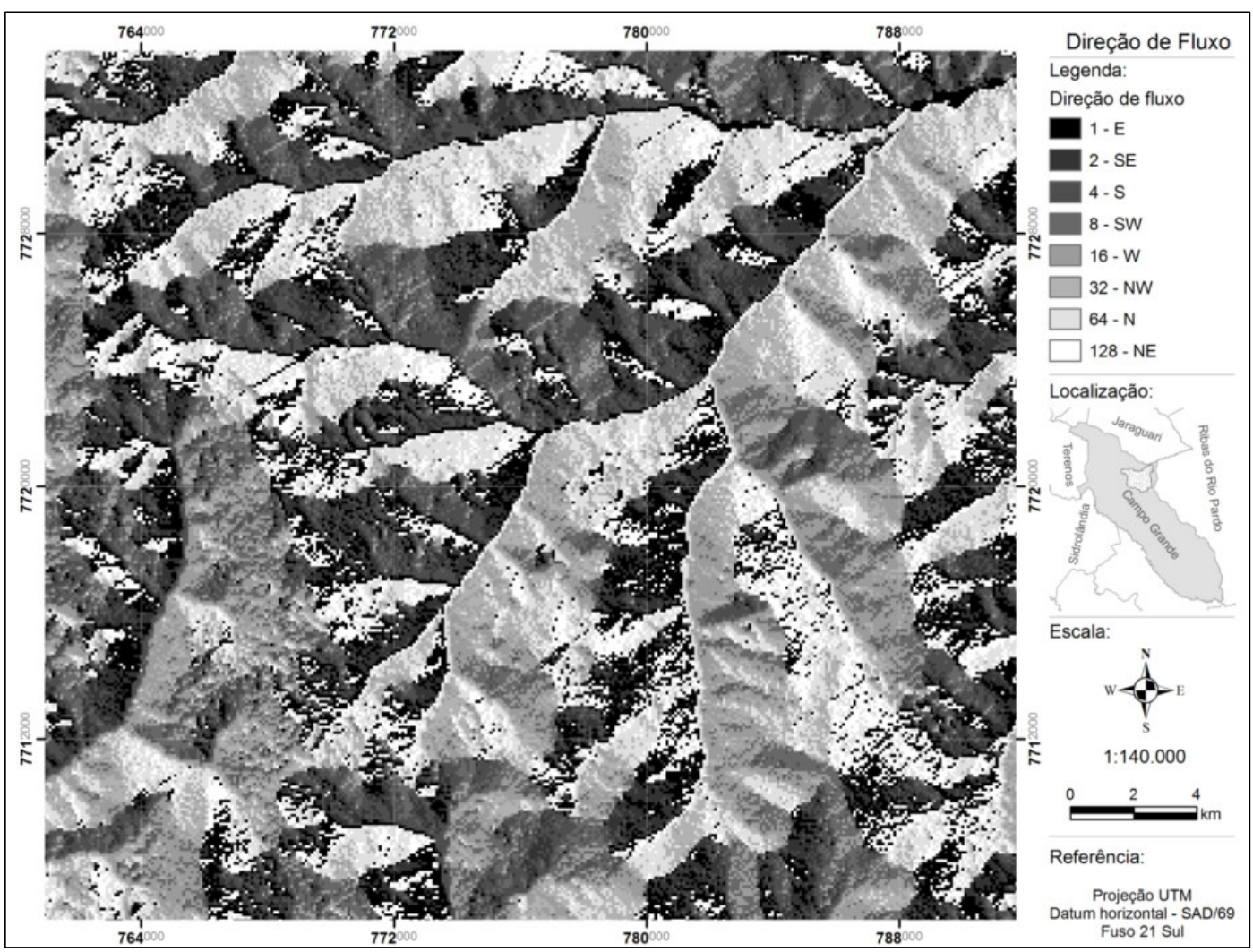

FIGURA 6. Mapa de direção de fluxo. Map of flow direction.

\section{Fluxo acumulado}

O fluxo acumulado é um parâmetro que indica o grau de confluência do escoamento e pode ser associado ao fator comprimento de rampa aplicado em duas dimensões. O fluxo acumulado, também denominado área de captação, apresenta obtenção complexa, manual ou computacional, uma vez que reúne, além de características do comprimento de rampa (conexão com divisores de água a montante), também a curvatura horizontal (confluência e divergência das linhas de fluxo) (VALERIANO, 2008).

Na Figura 7, exemplifica-se o fluxo acumulado, sendo possível observar a formação dos caminhos preferenciais de fluxo que originam a rede hidrográfica.
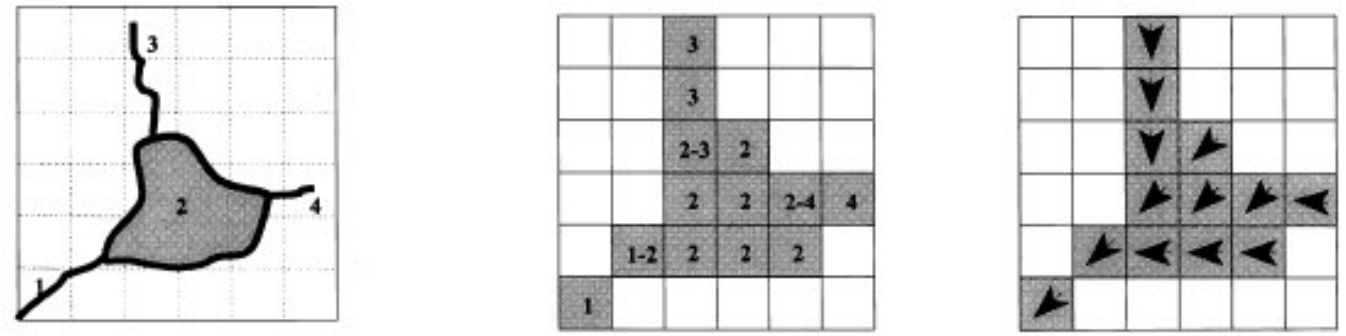

FIGURA 7. Exemplo de determinação do fluxo acumulado. Example of determining of accumulated flow. Fonte: Adaptado de TURCOTE et al. (2001). 
A partir da grade regular gerada, conforme descrito na etapa anterior, o fluxo acumulado foi obtido pela função "flow accumulation".

De acordo com MENDES \& CIRILO (2001), o fluxo acumulado representa a rede hidrográfica (Figura 8), sendo possível montar nova grade contendo os valores de acúmulo de água em cada "pixel". Desse modo, cada "pixel" recebe um valor correspondente ao número de "pixels" que contribuem para que a água chegue até ele.

A partir da direção de fluxo, o fluxo acumulado é obtido somando-se a área das células (quantidade de células) na direção do fluxo (ou escoamento).

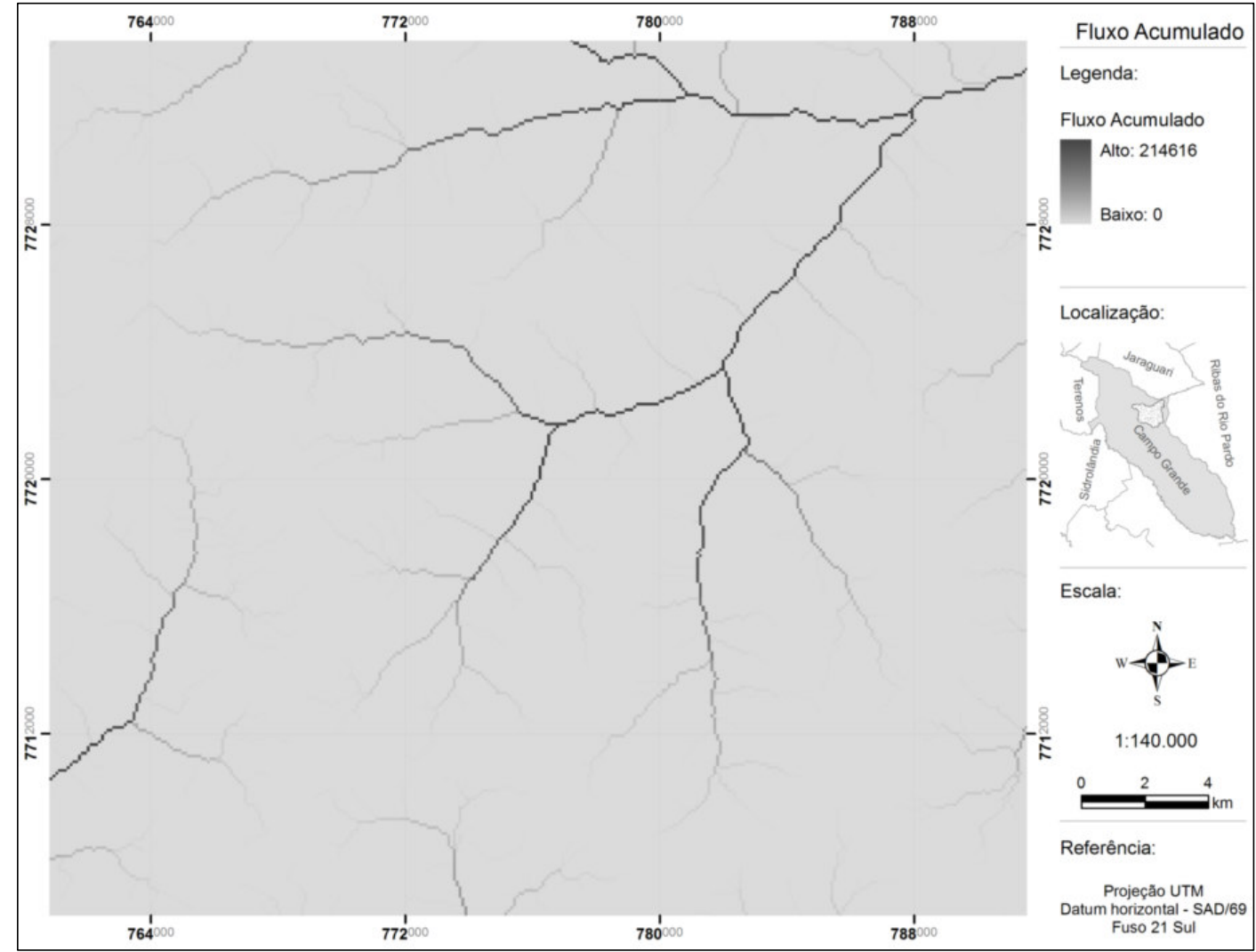

FIGURA 8. Mapa de fluxo acumulado. Map of accumulated flow.

\section{Delimitação de bacias}

O delineamento de microbacias é favorecido, significantemente, pelo processo denominado por VALERIANO (2008) como ADD que, em essência, é uma sobreposição de fatiamentos criteriosos da curvatura horizontal (para realce da drenagem e divisores de água) a classes de orientação de vertentes.

As feições de drenagem e divisores de água, convertidas em vetores, são alvos de análises clássicas do terreno em que se busca a delimitação de zonas homólogas para fins de mapeamento geológico, geomorfológico e pedológico.

A delimitação das bacias é realizada processando os mapas de direção de fluxo e fluxo acumulado na função "Watershed".

$\mathrm{O}$ valor da área de cada bacia hidrográfica gerada corresponde à quantidade de células que são processadas. Como cada célula do MNT obtido do SRTM possui "pixels" de $90 \mathrm{~m}$, a área de cada "pixel" é equivalente a $8.100 \mathrm{~m}^{2}$. Desse modo, realizou-se a definição do valor de células, sendo 
realizados testes até obter a que melhor correspondesse à área de estudo. Quanto menores os valores de célula definidos, maior o número de microbacias é criado.

O valor definido para este trabalho foi de 45.000 células. Assim, foram processadas bacias de até $364.500 .000 \mathrm{~m}^{2}$, equivalente a 36.450 ha. Depois de processar as informações na função "Watershed", delimitaram-se as bacias, que, posteriormente, foram convertidas para o formato vetorial, na função "Raster to Features" da extensão "Spatial Analyst".

Após a delimitação das bacias hidrográficas, foi utilizado o comando "Stream Network" para delimitação da rede hidrográfica, utilizando, para tanto, os resultados da direção de fluxo, fluxo acumulado, e adotando o valor 500 como o número mínimo de células para geração de fluxo.

\section{RESULTADOS E DISCUSSÃO}

O limite da área de drenagem extraída está representado na Figura 9, juntamente com as curvas de nível equidistantes de $90 \mathrm{~m}$ e a rede hidrográfica, geradas a partir dos dados SRTM.

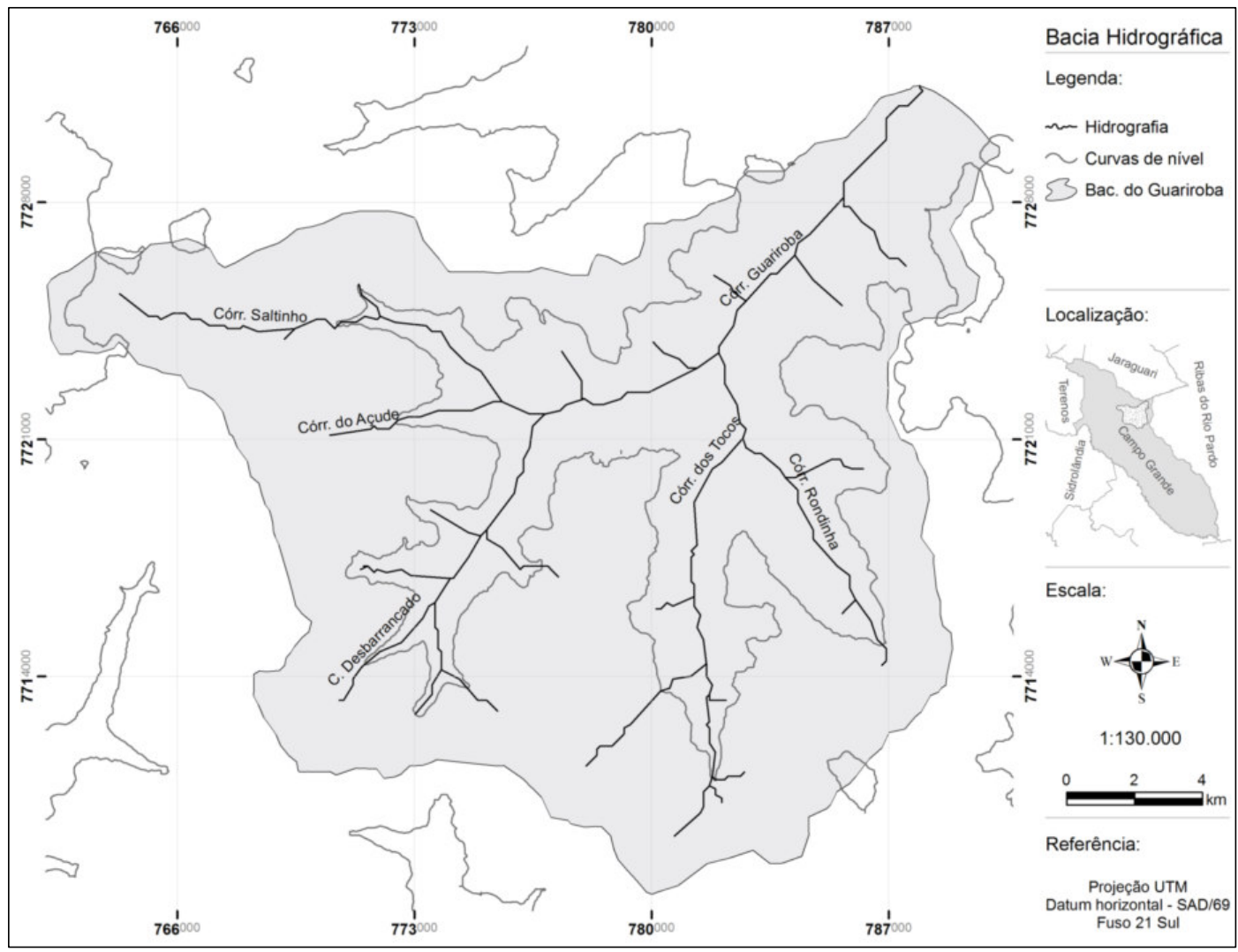

FIGURA 9. Bacia hidrográfica do Córrego Guariroba gerada a partir dos dados SRTM. Guariroba river basin generated from SRTM data.

A bacia hidrográfica do Córrego Guariroba, resultante da delimitação deste estudo, constitui área de $36.914,336$ ha e perímetro de $98,384 \mathrm{~km}$. Comparando-se com os resultados obtidos por TORRES et al. (2005) no mesmo local, verifica-se diferença, relativamente pequena, em relação à delimitação automática que utiliza cartas topográficas na escala 1:100.000 (Tabela 1). 
TABELA 1. Áreas delimitadas da bacia do Córrego Guariroba. Areas delimitated of Guariroba river basin.

\begin{tabular}{lccc}
\hline \multicolumn{1}{c}{ Base de Dados } & Metodologia & Área (ha)** & $\begin{array}{c}\text { Diferença em Relação à Base } \\
\text { de Dados do SRTM(\%) }\end{array}$ \\
\hline Carta topográfica & Manual & $36.110,564$ & 2,18 \\
(Escala 1:100.000) & Automática - Arc Info (ESRI) & $36.855,401$ & 0,16 \\
& Automática - Geomática (PCI) & $37.162,894$ & 0,66 \\
\hline
\end{tabular}

*TORRES et al. (2005)

TORRES et al. (2005) utilizaram, como referência, cartas topográficas elaboradas pela Diretoria do Serviço Geográfico (DSG), na escala 1:100.000, que exibem curvas de nível equidistantes de $40 \mathrm{~m}$, pontos cotados e hidrografia. Na metodologia utilizada pelos autores, para a geração do MNT, utilizado no processo de delimitação da bacia hidrográfica, foram necessários a digitalização, o georreferenciamento e a vetorialização das representações das curvas de nível, hidrografia e pontos cotados presentes em cada carta. Assim, nota-se que a metodologia utilizada neste estudo, baseada na utilização de dados do SRTM, mostra-se mais ágil e proporciona resultados compatíveis.

Observa-se que a delimitação automática de bacias tem uma subjetividade menor e que, mesmo utilizando "softwares" diferentes, os resultados são mais próximos do que quando comparados a métodos manuais que variam com a percepção humana. JENSON \& DOMINGUE (1988) e MERKEL et al. (2008), comparando as metodologias automática e manual, confirmam a eficiência do modo automático de extração da rede de drenagem a partir de MNT.

Pode-se afirmar que os dados do SRTM foram relativamente precisos e acurados, no caso analisado. Além de serem eficientes por demandarem menor tempo no processo de tratamento dos dados brutos até o delineamento da bacia, já que os dados do SRTM fornecem diretamente o MNT, ao passo que as cartas topográficas necessitam de procedimento inicial trabalhoso até a obtenção do MNT.

JARVIS et al. (2004) verificaram que cartas topográficas com escalas maiores que 1:25.000 (1:10.000, por exemplo) contêm aspectos topográficos que não são capturados pelo SRTM (resolução de $90 \mathrm{~m}$ ), obtendo-se, portanto, melhores resultados por meio da digitalização e da interpolação desses dados cartográficos para a geração dos MNTs. Em contrapartida, deve-se dar preferência aos dados do SRTM a cartas topográficas com escalas menores que 1:25.000 (1:50.000, por exemplo).

Considerando os resultados obtidos, avalia-se como adequada a metodologia proposta neste estudo a partir dos dados do SRTM e do aplicativo ArcGis. Portanto, vale ressaltar a possibilidade de aplicação dessa técnica a outras áreas, proporcionando, assim, a redução da subjetividade e dos erros provenientes do método manual de traçado de bacias hidrográficas.

\section{CONCLUSÕES}

A metodologia aplicada mostra-se adequada e de fácil utilização, podendo ser empregada a estudos em outras localidades.

A delimitação automática da rede de drenagem obtida a partir de dados do SRTM apresenta precisão compatível àquela baseada em cartas topográficas.

Fatores como gratuidade, precisão e acurácia dos dados do SRTM proporcionam economia de tempo e de recursos para a realização de pesquisas e diagnósticos ambientais apoiados nesses dados, especialmente em regiões que não possuem registros cartográficos.

A delimitação automática de bacias, por meio do processamento de dados do SRTM em ambiente SIG, apresenta-se vantajosa em relação ao custo e benefício proporcionado, além de 
estabelecer a padronização do traçado e posterior minimização de conflitos quanto à fixação da unidade elementar de gestão dos recursos hídricos.

\section{REFERÊNCIAS}

BAND, L.E. Topographic partition of watershed with digital elevation models. Water Resource Research, Washington, v.22, n.1, p.15-24, 1986.

BERRY, P.A.M.; GARLICK, J.D.; SMITH, R.G. Near-global validation of the SRTM DEM using satellite radar altimetry. Remote Sensing of Environment, New York, v.106, n.1, p.17-27, 2007.

BRASIL. Ministério das Minas e Energia. Projeto RADAMBRASIL. Folha SF.21 Campo Grande: geologia, geomorfologia, pedologia, vegetação e uso potencial da terra. Levantamento de Recursos Naturais, 28. Rio de Janeiro, 1982. 412 p.

DIAS, L.S.O.; ROCHA, G.A.; BARROS, E.U.A.; MAIA, P.H.P. Utilização do radar interferométrico para delimitação automática de bacias hidrográficas. Bahia Análise \& Dados, Salvador, v. 4, n.2, p.265-271, 2004.

ESRI. ENVIRONMENTAL SYSTEMS RESEARCH INSTITUTE, ArcGIS Professional GIS for the desktop, version 9.2. Software. 2006.

ESRI. ENVIRONMENTAL SYSTEMS RESEARCH INSTITUTE. ArcGIS 9.2 Desktop help. Disponível em:

$<$ http://webhelp.esri.com/arcgisdesktop/9.2/index.cfm?TopicName=Flow_Direction>. Acesso em: 8 ago. 2008.

FAIRFIELD, J.; LEYMARIE, P. Drainage networks from grid digital elevation models. Water Resources Research, Washington, v.27, n.5, p.709-717, 1991.

FALORNI, G.; TELES, V.; VIVONI, E.R.; BRAS, R.L.; AMARATUNGA, K.S. Analysis and characterization of the vertical accuracy of digital elevation models from the Shuttle Radar Topography Mission. Journal of Geophysical Research, Washington, v.110, n.F2, p.1-20, 2005.

FREDRICK, K.C.; BECKER, M.W.; MATOTT, L.S.; DAW, A.; BANDILLA, K.; FLEWELLING, D.M. Development of a numerical groundwater flow model using SRTM elevations. Hydrogeology Journal, Heidelberg, v.15, n.1, p.171-181, 2007.

GERSTENECKER, C.; LÄUFER, G.L.; STEINECK, D.; TIEDE, C.; WROBEL, B. Validation of digital elevation models around Merapi Volcano, Java, Indonesia. Natural Hazards and Earth System Sciences, Perugia, v.5, n.6, p.863-876, 2005.

JARVIS, A.; RUBIANO, J.; NELSOM, A.; FARROW, A.; MULLIGAN, M. Practical use of SRTM data in the tropics - comparisons with digital elevation models generated from cartographic data. International Center for Tropical Agriculture, Palmira, n.198, p.1-32, 2004.

JENSON, S.K.; DOMINGUE, J.O. Extracting topographic structure from digital elevation data for geographic information system analysis. Photogrammetric Engineering and Remote Sensing, Bethesda, v.54, n.11, p.1.593-1.600, 1988.

JORDAN, G.; SCHOTT, B. Application of wavelet analysis to the study of spatial pattern of morphotectonic lineaments in digital terrain models. Remote Sensing of Environment, New York, v.94, n.1. p.31-38, 2005.

KÄÄB, A. Combination of SRTM3 and repeat ASTER data for deriving alpine glacier flow velocities in the Bhutan Himalaya. Remote Sensing of Environment, New York, v.94, n.4, p. 463474, 2005. 
KOCAK, G.; BUYUKSALIH, G.; ORUC, M. Accuracy assessment of interferometric digital elevation models derived from the Shuttle Radar Topography Mission X- and C-band data in a test area with rolling topography and moderate forest cover. Optical Engineering, Bellingham, v.44, n.3, 2005.

LUEDELING, E.; SIEBERT, S.; BUERKERT, A. Filling the voids in the SRTM elevation model A TIN-based delta surface approach. ISPRS Journal of Photogrammetry \& Remote Sensing, New York, v.62, n.4, p.283-294, 2007.

MARK, D.M. Automatic detection of drainage networks from digital elevation models. Cartographica, Toronto, v.21, n.3, p.168-178, 1984.

MENDES, C.A.B.; CIRILO, J.A. Geoprocessamento em recursos hídricos: princípios, integração e aplicação. Porto Alegre: ABRH, 2001. 536 p.

MERKEL, W.H.; KAUSHIKA, R.M.; GORMAN, E. NRCS GeoHydro-A GIS interface for hydrologic modeling. Computers \& Geosciences, Oxford, v.34, n.8, p.918-930, 2008.

O'CALLAGHAN, J.F.; MARK, D.M. The extraction of drainage networks from digital elevation data. Computer Vision, Graphics, and Image Processing, San Diego, v.28, n.3, p.323-344, 1984.

PINHEIRO, E.S. Comparação entre dados altimétricos Shuttle Radar Topography Mission, cartas topográficas e GPS: Numa área com relevo escarpado. Revista Brasileira de Cartografia, Rio de Janeiro, v.58, n.1. p. 1-9, 2006.

RABUS, B.; EINEDER, M.; ROTH, A.; BAMLER, R. The shuttle radar topography mission: a new class of digital elevation models acquired by spaceborne radar. ISPRS Journal of Photogrammetry \& Remote Sensing, New York, v.57, n.4. p. 241-262, 2003.

RENNÓ, C.D.; NOBRE, A.D.; CUARTAS, L.A.; SOARES, J.V.; HODNETT, M.G.; TOMASELLA, J.; WATERLOO, M.J. HAND, a new terrain descriptor using SRTM-DEM: Mapping terra-firme rainforest environments in Amazonia. Remote Sensing of Environment, New York, v.112, n.9, p.3469-3481, 2008.

RODRIGUEZ, E.; MORRIS, C.S.; BELZ, J.E. A global assessment of the SRTM performance. Photogrammetric Engineering and Remote Sensing, Bethesda, v.72, n.3. p. 249-260, 2006.

SANTOS, P.R.A.; GABOARDI, C.; OLIVEIRA, L.C. Avaliação da precisão vertical dos modelos SRTM para a Amazônia. Revista Brasileira de Cartografia, Rio de Janeiro, v.58, n.1, p. 101-107, 2006.

SMITH, B.; SANDWELL, D. Accuracy and resolution of shuttle radar topography mission data. Geophysical Research Letters, Washington, v.30, n.9, p.1-4, 2003.

SUN, G.; RANSON, K.J.; KHAIRUK, V.I.; KOVACS, K. Validation of surface height from shuttle radar topography mission using shuttle laser altimeter. Remote Sensing of Environment, New York, v.88, n.4, p.401-411, 2003.

TARBOTON, D.G.; BRAS, R.L.; RODRIGUEZ-ITURBE, I. On the extraction of channel networks from digital elevation data. Hydrological Processes, Chichester, v.5, n.1, p.81-100, 1991.

TORRES, T.G.; PARANHOS FILHO, A.C.; RONDON, M.A.C.; LASTORIA, G.; SOUZA, A. Comparação do divisor de bacia obtido de diferentes modos: o caso de estudo da bacia do córrego Guariroba - MS. Revista de Estudos Ambientais, Blumenau, v.7, n.1. p. 39-56, 2005.

TURCOTTE, R.; FORTIN, J.P.; ROUSSEAU, A.N.; MASSICOTTE, S.; VILLENEUVE, J.P. Determination of the drainage structure of a watershed using a digital elevation model and a digital river and lake network. Journal of Hydrology, Amsterdam, v.240, n.3, p.225-242, 2001.

VALERIANO, M.M. Dados topográficos. In: FLORENZANO, T.G. (Org.). Geomorfologia, conceitos e tecnologias atuais. São Paulo: Oficina de Textos, 2008. p.72-104. 
VALERIANO, M.M.; ABDON, M.M. Aplicação de dados SRTM a estudos do Pantanal. Revista Brasileira de Cartografia, Rio de Janeiro, v.59, n.1, p.63-71, 2007.

VERDIN, K.L.; VERDIN, J.P. A topological system for delineation and codification of the Earth's river basins. Journal of Hydrology, Amsterdam, v.218, n.1, p.1-12, 1999.

VOGT, J.V.; COLOMBO, R.; BERTOLO, F. Deriving drainage networks and catchment boundaries: a new methodology combining digital elevation data and environmental characteristics. Geomorphology, Amsterdam, v.53, n.3, p.281-298, 2003.

WALKER, J.P.; WILLGOOSE, G.R. On the effect of DEM accuracy on hydrology and geomorphology models. Water Resource Research, Washington, v.357, n.7, p.2.259-2.268, 1999. 